\title{
Optical properties of HgTe colloidal quantum dots.
}

\section{Emmanuel Lhuillier, Sean Keuleyan, and Philippe Guyot-Sionnest ${ }^{1}$}

James Franck Institute, 929 E. 57 $7^{\text {th }}$ Street, The University of Chicago, Chicago, Illinois 60637,

\section{USA}

Abstract: Room temperature photodetection with $\mathrm{HgTe}$ colloidal quantum films is reported between 2 and 5 microns for particles of sizes between $\sim 5$ and $\sim 12 \mathrm{~nm}$ diameter, and photodetection extends to 7 microns at $80 \mathrm{~K}$. The size tuning of the absorption of $\mathrm{HgTe}$ colloidal quantum dots, their optical cross-section and the infrared absorption depth of films are measured. The tuning with radius is empirically given by $\lambda_{B E}^{Q D}=\frac{30.5}{\sqrt{1+\left(43 / R_{Q D}\right)^{2}}-1}$ where $\mathrm{R}$ is in $\mathrm{nm}$. The optical cross-section of the colloidal dots at $415 \mathrm{~nm}$ is approximately proportional to their volume and given by $\sigma_{H g}^{415}=2.6 \pm 0.4 \times 10^{-17} \mathrm{~cm}^{2}$ per mercury atom. The size-dependent optical cross-section at the band edge $\sim 1.5 \times 10^{-15} \mathrm{~cm}^{2}$, is consistent with the expected oscillator strength of the quantum dots. The absorption depth of HgTe colloidal dot films is short, about 1 to 2 microns, which is an advantage for thin film devices. These properties agree rather well with the expectation from the $\mathrm{k} \cdot \mathrm{p}$ model. $\mathrm{HgTe}$ colloidal quantum dot thin films show a strong tuning with temperature with a large positive thermal shift between $0.4 \mathrm{meV} / \mathrm{K}$ and 0.2 $\mathrm{meV} / \mathrm{K}$ decreasing with decreasing size within the size range studied and this is attributed primarily to electron-phonon effects.

Keywords: HgTe, quantum dot, band structure, polaron

\footnotetext{
${ }^{1}$ To whom correspondence should be sen: pgs@uchicago.edu
} 
Colloidal nanomaterials are widely investigated for their potential in electronic and optical applications ${ }^{[1]}$. Colloidal quantum dots (CQDs) provide specific opportunities with early developments focusing on the visible spectrum, primarily with the highly monodisperse cadmium chalcogenides ${ }^{[2]}$. In the past decade, the near-infrared has become of interest, with the most studied materials being the lead chalcogenides ${ }^{[3]}$, lately motivated largely by the hope of creating cheap photovoltaic thin films ${ }^{[4,5]}$. With CQDs of the appropriate inorganic materials, the optical response can be extended into the mid-infrared and this may lead to a lower cost alternative to the currently expensive infrared imaging technology. The mercury chalcogenides with a zero bulk band gap fulfill this opportunity and $\mathrm{HgTe} C Q D$ films have recently enabled photodetection in the mid-IR ${ }^{[6,7,8]}$. $\mathrm{HgTe}$ colloids have been synthesized for more than a decade but the materials remained restricted to the near-infrared with broad absorption edges. ${ }^{[9,10,11,12]}$ Recently, more monodisperse HgTe colloidal dots have been synthesized ${ }^{[8]}$ providing materials with excitonic transitions well into the mid-infrared. In this work we follow ref. [8] and study the optical properties of these new materials. As previously reported in ref. [8], Figure 1 shows that the spectral photocurrent response of thin films of these HgTe CQD films is widely tunable with different sizes of particles. In addition, Figure 1 shows that the spectral response also tunes with temperature, showing a strong redshift with decreasing temperature. With these new colloidal semiconductor materials, we characterize some of the important optical properties, such as the size-tuning, the optical cross-sections in solution and the absorption depth of the films. The temperature tuning of the absorption is also of practical importance for detection, and we investigate the shift and its size dependence. The measured properties of the materials are compared to expectations based on the $\mathrm{k} \cdot \mathrm{p}$ model of bulk $\mathrm{HgTe}$.

$\mathrm{HgTe}$ colloidal dots are synthesized following reference [8]. Briefly, $\mathrm{HgCl}_{2}$ and oleylamine are heated to $100^{\circ} \mathrm{C}$ under vacuum for 1 hour. The mixture is then heated or cooled to the appropriate growth temperature for the target size (with higher temperature giving larger particles) and Te dissolved in trioctylphosphine is quickly added. Growth is stopped by quenching the solution into dodecanethiol in tetrachloroethylene and the particles purified by precipitation with methanol. Typical growth times are between 1 and $90 \mathrm{~min}$, with longer times giving larger particles. Various shapes including spheres, tetrahedra or even multipods are obtained, as shown in Figure 2 (a). In spite of the variety of morphology, the HgTe CQDs are always zinc blende, shown by X-ray diffraction (XRD) data in Figure 2 (b). The particle sizes studied here range from 5 to $12 \mathrm{~nm}$ as determined by TEM, which results in 
the band-edge energy tuning from the near to mid-IR. The size tuning of the absorption edge is shown in Figure 2(c).

The sizes in Figure 2 (c) were determined from TEM images as an effective diameter. Due to the significant variation in particle shape and the tendency of the particles to aggregate, we used XRD to obtain another measure of size, using the Debye-Scherrer formula as discussed in the Supplementary Information.

The optical cross-sections of colloidal dots are useful to determine the concentrations of colloidal solutions but they have not been reported previously for $\mathrm{HgTe}$ dots. The cross-sections reported here are obtained by first measuring the absorption in the UV-visible and infrared and determining the QD size from XRD. Then the samples are acid digested and the mercury content is measured by titration with dithizone. This procedure is described in detail in the Supplementary Information. As shown in Figure 3 (a), we found that the cross section of the $\mathrm{HgTe}$ QDs per $\mathrm{Hg}$ atom at $415 \mathrm{~nm}$ is almost constant over 6 sizes of nanoparticles. We therefore propose to use $\sigma_{\mathrm{Hg}}^{415}=2.6 \pm 0.4 \times 10^{-17} \mathrm{~cm}^{2}$ to directly estimate the number of $\mathrm{Hg}$ atoms. Using the size determined from the XRD data to estimate the number of $\mathrm{Hg}$ atoms per particle, and the optical densities at $415 \mathrm{~nm}$ and at the first exciton peak or plateau, we get the band-edge particle cross-section, $\sigma_{Q D}^{B E}$. The band-edge particle cross-section is typically around $1.5 \times 10^{-15} \mathrm{~cm}^{2}$.

From sample to sample, the band edge cross-section is affected by the size dispersion and the width of the exciton peak. This can be circumvented by using the integrated cross-sections $\sigma_{Q D}^{\mathrm{int}}$ which may then be compared to theoretical oscillator strengths. To get the integrated cross-section, the band edge is fitted to a Gaussian, giving $\sigma_{Q D}^{\mathrm{int}}=\sqrt{2 \pi \gamma_{\text {gauss }}^{2}} \sigma_{Q D}^{B E}$, where $\gamma_{\text {gauss }}$ is the Gaussian standard deviation in $\mathrm{cm}^{-1}$ determined for each sample. The results are shown in Figure 3 (b). The integrated cross section are in the $10^{-12} \mathrm{~cm}$ range with rather large error bars resulting from the uncertainty in the value of $\sigma_{H g}^{415}$, the uncertainty in the optical density at the band-edge, and the uncertainty in the volume of a quantum dot extracted from the XRD width (see supplementary information for more details). 
For the absorption measurements of films as well as for photodetection, the CQD are dispersed in hexane/octane (9:1) and drop-cast, leading to homogeneous films of $\sim 50 \mathrm{~nm}$ thickness. Then the films are immersed in a $1 \%$ solution of ethanedithiol in ethanol for $1 \mathrm{~min}$. As the bulky ligands are exchanged with shorter ones, the molecular vibrational absorption is reduced, the absorption slightly red-shifted, the thickness of the film decreases and the optical dielectric constant increases. To make thicker films, the process is repeated several times. For absorption, the films are drop cast on polished silicon wafers and their thickness is measured using an ellipsometer (Gartner L116S), while the film optical density is measured using an FTIR (Nicolet magna IR 550), see the inset of Figure 3 (c). The optical absorption depth of the material is an important parameter for infrared detection. With selfassembled epitaxial quantum dots, a long standing problem has been the low volume fraction which results in a small absorption coefficient. Here the close-packed colloidal dots give short absorption depths between 1 and 2 microns as shown in Figure 3 (c). The absorption coefficient of the CQD material is in fact similar to the one reported for bulk $\mathrm{HgTe}\left(5 \times 10^{3} \mathrm{~cm}^{-1}\right)^{[13]}$ and $\mathrm{HgCdTe}$ alloys ${ }^{[14,15,16]}$ with the same cut off wavelength, while the liquid processing of the colloidal quantum dots is a major advantage.

For photoconduction measurements, the films are drop-cast on to interdigitated planar Pt electrodes with 50 periods, $10 \mu \mathrm{m}$ spacing and $5 \mathrm{~mm}$ length (ABTech IME 1050). The photocurrent spectra are acquired with a Nicolet Magna IR 550 FTIR and are normalized to the response of a DTGS detector. To investigate the effect of the temperature on the spectra, the samples are mounted on the cold finger of a closed cycle He cryostat. Photocurrent spectra for different sizes and temperatures are presented in Figure 1. Figure 4 (a) shows the thermal shift of the band edge energy extracted from the photocurrent spectra shift. Using the empirical Varshni expression $E_{G}(T)=E_{G}(T=0 K)+\frac{\alpha T^{2}}{\beta+T}$, the data are fit with a value of $\beta$ of $160 \mathrm{~K}$ which is close to the bulk Debye temperature $\left(120 \mathrm{~K}^{[13]}\right)$. The slope in the higher temperature range gives the coefficient $\alpha$, which is found to be size dependent as shown in Figure 4(b). Figure 4 (b) also shows an empirical fit for $\mathrm{HgCdTe}$ alloys as a function of the bandgap ${ }^{[17]}$ and it is observed that the $\alpha$ parameter as a function of bandgap for HgTe CQDs follows trend similar to that of $\mathrm{HgCdTe}$. Extrapolating to zero bandgap from our data on $\mathrm{HgTe}$ CQDs gives +460 $\mu \mathrm{eV} . \mathrm{K}^{-1}$, which is close to the expected bulk value for HgTe, seen in Figure 4 (b) as the y-intercept of the HgCdTe 
data. This is also within the range of other reported values, 270 to $850 \mu \mathrm{eV} . \mathrm{K}^{-1}$, see ref [18] and [19]. The trend of smaller $\alpha$ with larger bandgap CQDs is similar to $\mathrm{PbS}^{[3]}$ and $\mathrm{PbSe}^{[20]} \mathrm{CQDs}$ but is in contrast with CdSe CQDs, which show a thermal red-shift $\left(\frac{d E_{B E}^{Q D}}{d T}<0\right)$, independent of CQD size ${ }^{[21]}$.

Early work comparing small $\mathrm{HgTe}$ quantum dots with calculations based on the effective mass and the negative band gap, predicted the $\Gamma_{6}$ and $\Gamma_{8}$ gap but not the optical gap. ${ }^{[10]}$ More recently k•p calculations of $\mathrm{HgTe}$ spherical nanocrystals have been reported, ${ }^{22}$ and include the mixing of the bands in the envelope functions. In Figure $2 \mathrm{c}$, a comparison with our experimental results indicates that ref. [22] vastly overestimates the energies in particular for the smaller sizes. In our k•p calculations, we follow the parametrization of ref. [23] The $8 \times 8 \mathrm{k} \cdot p$ matrix leads to the numerically calculated band diagram shown in Figure 5 for the range of k-vectors relevant to the particles in this study. Parameters used are summarized in Table 1 . The photonic transition takes place between the two $\Gamma_{8}$ bands in the HgTe CQD. Figure 2 (c) (solid line) shows the expected tuning of the first exciton with size for a spherical HgTe particle where the momentum, $k$, of electron and hole wavefunctions are given by $k=\pi / R_{Q D}$ with $R_{Q D}$ the nanoparticle radius. This neglect of band mixing in the envelope function should be appropriate in the limit of infinite confinement potential and nondegenerate band structure. The $k \cdot p$ size-tuning that we obtain is in fair agreement with the experimental data within the errors in the size determination. The discrepancy with ref. [22] might be due to the use of different parameters. In particular ref. [22] used $E_{G}=-0.3 e V$ while we use the room temperature value of $E_{G}=-0.15 e V[23]$.

For practical use, we provide empirical relations between the band gap (in $\mu \mathrm{m}$ ) and the particle size (in $\mathrm{nm}$ ),

$$
\left.\lambda_{B E}^{Q D}=\frac{30.5}{\sqrt{1+\left(43 / R_{Q D}\right)^{2}}-1}\right) \text { and } R_{Q D}=\frac{43}{\sqrt{\left(1+30.5 / \lambda_{B E}^{Q D}\right)^{2}}-1}
$$

The choice of this fitting formula is based on the underlying $k \cdot p$ relations discussed below and provides a rational trend at small and large sizes - trends that would be missed in a multiparameter polynomial fit. The expression arises from simplifying further the $\mathrm{k} \cdot \mathrm{p}$ by considering that, the spin-orbit of $\mathrm{HgTe}$ is very large, and that $\mathrm{k} \cdot \mathrm{p}$, restricted to the two bands $\Gamma_{6}$ and $\Gamma_{8},{ }^{[24]}$ already captures the size/energy relationship rather well. The two-band $k \cdot p$ gives the light hole and conduction band as 


$$
E_{C / L H}=-\frac{E_{G}}{2} \pm \sqrt{\frac{E_{G}^{2}}{4}+\frac{2}{3} E_{P} \frac{\hbar^{2} k^{2}}{2 m_{0}}}
$$

with $\mathrm{m}_{0}$ the free electron mass, $\hbar$ the reduced Planck constant, $E_{P}$ the Kane parameter, $E_{G}$ the bulk band gap $\left(\mathrm{E}_{\Gamma_{6}}-\mathrm{E}_{\Gamma_{8}<0}\right)$ and the heavy hole energy is taken as non dispersive

$$
E_{H H}=0
$$

The band edge transition is between the heavy hole and the conduction band and its energy is simply

$$
E_{B E}^{Q D}=\frac{E_{G}}{2}+\sqrt{\frac{E_{G}^{2}}{4}+\frac{2}{3} E_{P} \frac{\hbar^{2} k^{2}}{2 m_{0}}}
$$

This two-band analytical expression is the basis for the form used above as an empirical fit in equation 1. We are not aware of previous comparisons between theoretical and experimental absorption strengths for $\mathrm{HgTe}$ nanocrystals. Therefore we use the two-band $k \cdot p$ model to analytically get the oscillator strength as

$$
f=\frac{E_{P}}{2 E_{B E}}\left(\frac{E_{c}^{2}}{2 / 3 E_{P} \frac{\hbar^{2} k^{2}}{2 m_{0}}}+1\right)^{-1}
$$

$f$ varies from 5 to 10 in the size range studied here. The integrated (over wavenumber) absorption cross-section of the first exciton (with $k=\pi / R_{Q D}$ ) is

$$
\sigma_{Q D}^{\mathrm{int}}=2 \frac{e^{2}}{4 \varepsilon^{\circ} m_{0} c^{2} n} S f
$$

where $\mathrm{n}$ is the optical index of the solution ( $\mathrm{n} 1.5$ for TCE). $S$ is the screening factor, $S=\left(\frac{3 \varepsilon_{1}}{\varepsilon_{2}+2 \varepsilon_{1}}\right)^{2}$, where $\varepsilon_{1}$ and $\varepsilon_{2}$ are the dielectric constants of the medium (TCE, 2.25) and $\mathrm{HgTe}$ (labeled $\varepsilon_{\infty}$ in Table 1) respectively, $\varepsilon^{\circ}$ the vacuum permittivity. The factor of two in front of equation (6) accounts for the two electrons that lead to the first absorption in the QD. Figure 3 (b) shows the calculated integrated cross-sections, which are in fair agreement with the experimental data. 
To estimate a theoretical absorption coefficient for the films, we use the integrated cross-section expression in equation (6). We assume a FWHM of $15 \%$ which is consistent with the width of the band edge absorption, and get a peak cross-section $\sigma_{\text {peak }}$. The film absorption coefficient is then taken as $\alpha_{a b s}=\sigma_{\text {peak }} \frac{\eta}{V_{Q D}}$ where $V_{Q D}$ is the volume of the particle. $\eta$ is the filling factor of the film by the CQD taken equal to 0.64 assuming a random close packing of the CQDs. We neglect the effects of reflectivity on the internal field but account for the microscopic local field using the film optical index determined by ellipsometry at $632 \mathrm{~nm}$ to be $2.5 \pm 0.1$ after crosslinking. The calculated absorption depth shown in Figure 3 (c) is in fair agreement with the measured absorption. Overall, we then conclude that the $k \cdot p$ method gives a fair description of the size-tuning of the absorption edge of the nanoparticles, as well as the strength of the optical absorption.

We now turn our attention to the thermal shift of the absorption edge and its size dependence shown in Figure 4 (b). A possible origin of the size dependence is the change of the band structure as the gap energy changes. Figure 5 shows the $\mathrm{k} \cdot \mathrm{p}$ bulk bands at 80 and $300 \mathrm{~K}$ with the empirical value of the gap at that temperature. Figure 5 shows that $k \cdot p$ leads to a red shift with decreasing temperature of the right order of magnitude but wrongly predicts an increase in the thermal shift with smaller sizes (larger k), in contradiction to observations. There is no reasonable choice of parameters that could reverse the trend. Since the size dependence of the temperature effect is not accounted for by simply changing the bulk bandgap parameter in the $k \cdot p$ model, one needs to look at the source of the thermal shift.

In general, the thermal shift of the gap is assigned to electron-phonon effects and thermal lattice expansion such that ${ }^{[25]} \Delta E_{g}(T)=\Delta E_{g}^{e l-p h}(T)+\Delta E_{g}^{\text {exp }}(T)$. The thermal shift for CQDs can then be discussed by starting from the bulk properties and including the effect of confinement.

Thermal expansion: The effect of lattice thermal expansion is given by the deformation potential, $\mathrm{D}$, and the lattice expansion, $\alpha_{d i l}$, such that $\frac{\Delta E_{g}^{\exp }}{\Delta T} \sim 3 \alpha_{d i l} D^{[25]}$. For HgTe this is a red-shift of $-28 \mu \mathrm{eV} \mathrm{K}^{-1}$ which is small 
compared with the bulk bandgap shift, $+460 \mu \mathrm{eV} \mathrm{K}^{-1}$, such that we can conclude that lattice expansion plays a negligible role in the thermal shift of $\mathrm{HgTe} C Q D s$.

Expansion of the lattice also changes the size of the particle, which in turn will affect the band gap energy as $\frac{\Delta E_{B E}^{Q D}}{E_{B E}^{Q D}} \sim-2 \frac{\Delta R_{Q D}}{R_{Q D}}=-2 \alpha_{d i l} \Delta T$, leading to $\frac{\Delta E_{B E}^{Q D}}{\Delta T}=-2 \alpha_{d i l} E_{B E}^{Q D}$. With a $0.3 \mathrm{eV}$ band edge as an example, the envelope expansion contributes only $-3 \mu \mathrm{eV} \cdot \mathrm{K}^{-1}$ which is of totally negligible magnitude. Therefore, the lattice expansion does not account for the observed trend and magnitude of the size dependent thermal shift in $\mathrm{HgTe}$ CQDs.

Electron-phonon effects: The remaining cause can be a change of the band edge energy due to electron-phonon effects. At constant volume, the energy of the electron wavefunction changes with temperature due to the interaction with phonons and depends on the electronic and phonon density of states. While the literature varies in the assessment of the accuracy of the electron-phonon calculation in accounting for the bulk thermal shift of the mercury chalcogenides, ${ }^{[17,18,19]}$ it is in fact apparent that it is the dominant contribution. The electron-phonon coupling $H_{e-p h}$, treated in second order perturbation theory, leads to an energy shift of an occupied electronic state $n$ of the general form ${ }^{[26]}$

$$
\Delta E_{n}=-\sum_{n, m^{\prime}} \frac{\left|\left\langle n^{\prime}, m \pm 1\left|H_{e-p h}\right| n, m\right\rangle\right|^{2}}{E_{n^{\prime}} \mp \hbar \omega_{p h}-E_{n}}
$$

Where the phonon energy is $\hbar \omega_{p h}, n^{\prime}$ are empty states and $m$ is a particular phonon occupation. For optical phonons, which are expected to dominate in the polar $\mathrm{HgTe}$ material, the coupling in the numerator in (1) has been shown to have a weak size dependence ${ }^{[27,28]}$. However, the denominator in equation (7) increases with confinement, which will reduce the electron-phonon effect. This suggests therefore that, for HgTe CQDs, the dominant contribution to the reduced thermal shift with decreasing sizes is the reduction of the electron-phonon interaction at large confinement. The experimental data presented here on HgTe CQDs should generate further interest in calculating the effect. 
In summary, this paper reports a first investigation of several optical properties of $\mathrm{HgTe}$ colloidal quantum dots tuning from 2 to 5 microns at room temperature. The size-tuning and the optical cross-section of the colloidal solutions are reported. Films have also a strong optical absorption, suitable for thin film photodetectors. The observations agree rather well with calculations using the $k \cdot p$ model. The photodetection range of $\mathrm{HgTe}$ colloidal quantum dots thin films tunes with temperature and the shift is significant. For example, the detection cut-off tunes from $5 \mu \mathrm{m}$ at room temperature to $7 \mu \mathrm{m}$ at low temperature for the largest particles studied here. The magnitude of the shift decreases at small sizes and this is suggested to arise from the reduced electron-phonon coupling at higher confinement energy.

Acknowledgements: This work was supported by the DARPA COMPASS program through a grant from ARO.. 
Figure 1 Normalized photocurrent spectra for three different sizes of HgTe nanoparticle at $T=3 K$ (dashed line) and at room temperature (solid line). The particle sizes are respectively $5 \mathrm{~nm}$ (bluest sample), $8 \mathrm{~nm}$ and $12 \mathrm{~nm}$ (reddest sample)

Figure 2 (a) TEM images of HgTe nanoparticles of different size and shape. (b) XRD data of a film of HgTe nanoparticles. (c) Band edge wavelength as a function of particle size for spherical particles. The solid curve is the result obtained though the $k \cdot p$ calculation. For the experimental points, the particle size is estimated from transmission electronic microscopy. The dashed curve is the $k \cdot p$ result from reference 22

Figure 3: (a) Cross-section per mercury atom as a function of particle size at $415 \mathrm{~nm}$. The dashed line is the mean value (b) Particle band edge integrated cross-section as a function of band edge energy. The solid line is the $k \cdot p$ calculation. (c) Absorption coefficient of films of HgTe particles as a function of the cut-off wavelength. The solid line is the result of the $k \cdot p$ calculation and the experimental points are indicated. The inset shows an absorption spectrum of a $60 \mathrm{~nm}$ thick film.

Figure 4 (a) Evolution of the band edge energy as a function of temperature for the three samples. The band edge energy is taken as the point of half-height of the rising edge. Dots are the experimental points and solid lines are the Varshni's fit (b) a Varshni parameter as a function of the energy band edge of the CQD. A linear fit of the curve is also proposed to obtain an extrapolation of the bulk value (i.e. null band gap). The dotted line is $\frac{d E_{G}}{d T}$ as a function of the band gap value for HgCdTe with different Cd composition ${ }^{[29]}$.

Figure 5 : (a) Band diagram for bulk HgTe using the bulk band-gap at two different temperatures (300K and 80K). 
Table 1 of the HgTe parameters used for modeling

\begin{tabular}{|c|c|c|}
\hline Quantity & Value & Reference \\
\hline $\begin{array}{l}E_{G}^{\text {bulk }}(T) \\
\text { Bulk band gap }\end{array}$ & $-303+\frac{0.63}{11+T} m e V$ & $\begin{array}{l}\text { Erreur! } \\
\text { Signet } \\
\text { non } \\
\text { défini. }\end{array}$ \\
\hline $\begin{array}{l}E_{P} \\
\text { Kane Energy }\end{array}$ & $18 \mathrm{eV}$ & $\begin{array}{l}\text { Erreur! } \\
\text { Signet } \\
\text { non } \\
\text { défini. }\end{array}$ \\
\hline $\begin{array}{l}\Delta_{S O} \\
\text { spin orbit coupling }\end{array}$ & $1 \mathrm{eV}$ & $\begin{array}{l}\text { Erreur! } \\
\text { Signet } \\
\text { non } \\
\text { défini. }\end{array}$ \\
\hline $\begin{array}{l}A^{\prime} \\
k p \text { modeling coefficient }\end{array}$ & $-9.08 \times 10^{-39} \mathrm{~J}^{2} \mathrm{~s}^{2} \mathrm{Kg}^{-1}$ & $\begin{array}{l}\text { Erreur! } \\
\text { Signet } \\
\text { non } \\
\text { défini. }\end{array}$ \\
\hline $\begin{array}{l}L^{\prime} \\
\text { kp modeling coefficient }\end{array}$ & $-2.97 \times 10^{-38} \mathrm{~J}^{2} \mathrm{~s}^{2} \mathrm{Kg}^{-1}$ & $\begin{array}{l}\text { Erreur! } \\
\text { Signet } \\
\text { non } \\
\text { défini. }\end{array}$ \\
\hline $\begin{array}{l}M \\
k p \text { modeling coefficient }\end{array}$ & $-2.48 \times 10^{-38} \mathrm{~J}^{2} \mathrm{~s}^{2} \mathrm{Kg}^{-1}$ & $\begin{array}{c}\text { Erreur! } \\
\text { Signet } \\
\text { non } \\
\text { défini. }\end{array}$ \\
\hline $\begin{array}{l}N^{\prime} \\
k p \text { modeling coefficient }\end{array}$ & $3.21 \times 10^{-38} \mathrm{~J}^{2} \mathrm{~s}^{2} \mathrm{Kg}^{-1}$ & $\begin{array}{l}\text { Erreur! } \\
\text { Signet } \\
\text { non } \\
\text { défini. }\end{array}$ \\
\hline $\begin{array}{l}\alpha_{d i l} \\
\text { Linear thermal expansion } \\
\text { coefficient }\end{array}$ & $5 \times 10^{-6} \mathrm{~K}^{-1}(300 \mathrm{~K})$ & 13,30 \\
\hline $\begin{array}{l}\mathrm{B}_{0} \\
\text { bulk modulus }\end{array}$ & $42.3 \mathrm{GPa}$ & 30,31 \\
\hline $\mathrm{B}_{1}$ & 4 & 30,31 \\
\hline $\begin{array}{l}D \\
\text { deformation potential }\end{array}$ & $-5.6 \mathrm{eV}$ & 13 \\
\hline $\begin{array}{l}\varepsilon_{0} \\
\text { Static dielectric constant }\end{array}$ & 20 & 32 \\
\hline $\begin{array}{l}\varepsilon_{\infty} \\
\text { Dielectric constant at } \\
\text { optical frequency }\end{array}$ & 14 & 13 \\
\hline$\hbar \omega_{L O}$ & $17 \pm 3 \mathrm{meV}$ & 33,33 \\
\hline
\end{tabular}




\begin{tabular}{lcc}
\hline \hline LO phonon energy & & \\
\hline $\begin{array}{l}\rho \\
\text { the mass density }\end{array}$ & $8.1 \times 10^{3} \mathrm{kgm}^{-3}$ & 13 \\
\hline$C_{s}$ & $2.14 \times 10^{3} \mathrm{~ms}^{-1}$ & 34 \\
The speed of sound & $0.23 \mathrm{Cm}^{-2}$ & 35 \\
\hline $\begin{array}{l}e_{14} \\
\text { piezoelectric constant }\end{array}$ & \\
\hline \hline
\end{tabular}




\section{REFERENCES}

${ }^{1}$ Talapin D., Lee J.S., Kovalenko M. and Shevchenko E. 2010 Chem. Rev. 110, 389.

2 Empedocles S A, Neuhauser R, Shimizu K, Bawendi M G 1999 Adv. Mat. 11, 1243.

${ }^{3}$ Olkhovets A, Hsu R-C, Lipovskii A, and Wise F W 1998 Phys. Rev. Lett. 81, 3539.

${ }^{4}$ Nozik A J, 2002, Physica E 14, 115.

${ }^{5}$ Kramer I J and Sargent E H 2011, ACS Nano 5, 8506.

${ }^{6}$ Keuleyan S, Lhuillier E, Brajuskovic V and Guyot-Sionnest P 2011 Nat. Photon. 5, 489.

${ }^{7}$ Lhuillier E, Keuleyan S, Rekemeyer P and Guyot-Sionnest P 2011 J. Appl. Phys 110, 032110.

${ }^{8}$ Keuleyan S, Lhuillier E and Guyot-Sionnest P 2011 J. Am. Chem. Soc. 133, 16422.

${ }^{9}$ Rogach A, Kershaw S, Burt M., Harrison M, Kornowski A, Eychmuller A, Weller H, Adv. Mater. 11, 552 (1999)

${ }^{10}$ Green, M, Wakefield, G, Dobson, PJ, 2003, J. Mat. Chem. 13, 1076-1078

${ }^{11}$ Kovalenko M. V., Kaufmann E., Pachinger D., RoitherJ., Huber M., Stangl J., Hesser G., Schäffler F., Heiss W., 2006 J. Am. Chem. Soc. 128, 3516.

${ }^{12}$ Hyuk Im S., Kim H.-j., Kim S. W, Kim S.-W. and Seok S. II, 2012 Nanoscale 4, 1581.

${ }^{13}$ Nimtz G and Schlicht B in Narrow gap semiconductors, Spinger tracts in modern Physics, vol 98, Berlin 1983.

${ }^{14}$ Blue M D 1964 Phys. Rev. 134, A226.

${ }^{15}$ Norton P 2002 Opto-Electronics Rev. 10, 159.

${ }^{16}$ Rogalski A 2005 Rep. Prog. Phys. 68, 2267.

${ }^{17} \mathrm{Nag}$ B R, Electron transport in compound semiconductors, Springer series in solid state sciences 11, Berlin 1980

${ }^{18}$ Guenzer C S and Bienenstock A 1973 Phys. Rev. B 8, 4655.

${ }^{19}$ Dobrowolska M, Mycielsji A and Dobrowolski W 1978 Solid Stat. comm. 27, 1233.

${ }^{20}$ Dai Q, Zhang Y, Wang Y, Hu M Z, Zou B, Wang Y and Yu W W 2010 Langmuir 26, 11435.

${ }^{21}$ Valerini D, Cretí A, Lomascolo M, Manna L, Cingolani R and Anni M 2005 Phys. Rev. B 71, 235409.

${ }^{22}$ Zhang XW and Xia J B, 2006 J. Phys. D: Appl. Phys. 39, 1815.

${ }^{23}$ Man P, and Pan D S 1991 Phys. Rev. B 44, 8745.

${ }^{24}$ Bastard G 1982 Phys. Rev. B 25, 7584 
${ }^{25}$ Franceschetti A 2007 Phys. Rev. B 76161301 (R).

${ }^{26}$ Weng C-L, Chen I-C and Tsai Y C 2007 Phys. Rev. B 76, 195313.

${ }^{27}$ Klein M C, Hache F, Ricard D and Flytzanis C 1990 Phys. Rev. B 42, 11123.

${ }^{28}$ Takagahara T 1993 Phys. Rev. Lett. 71, 3577.

${ }^{29}$ Hansen G L, Schmit J L, and Casselman T N 1982 J. Appl. Phys. 53, 7099.

${ }^{30}$ Besson J M, Grima P, Gauthier M, Itié J P, Mezouar M, Hausermann D and Hanfland M 1996 phys. stat. sol. (b) 198, 419.

${ }^{31}$ Latussek V, Becker C R, Landwehr G, Bini R and Ulivi L 2005 Phys. Rev. B 71, 125305.

${ }^{32}$ Baars J. and Sorger F.1972 Sol.State Comm. 10, 875.

${ }^{33}$ Kepa H, Giebultowicz T, Buras B., Lebech B and Clausen K 1982 Phys. Scr. 25, 807.

${ }^{34}$ Summers C J, Darling B and Martin B G 1986 J. Appl. Phys. 59, 2457.

${ }^{35}$ Varshney S C and Gundjian A A 1978 phys. Stat. sol (b) 85, 733. 